\title{
Antiatherogenic Effects of L-Arginine in the Hypercholesterolemic Rabbit
}

\author{
John P. Cooke, * Alan H. Singer, * Philip Tsao, * Pauline Zera, ${ }^{\star}$ Reed A. Rowan, ${ }^{\star}$ and Margaret E. Billingham ${ }^{*}$ \\ * Division of Cardiovascular Medicine and ${ }^{\ddagger}$ Department of Pathology, Stanford University School of Medicine, Stanford, California 94305
}

\begin{abstract}
The purpose of this study was to determine if chronic administration of L-arginine, the precursor of endothelium-derived relaxing factor (EDRF), normalizes endothelium-dependent relaxation and decreases atherosclerosis in hypercholesterolemic animals. Male rabbits were fed (a) normal rabbit chow; (b) $1 \%$ cholesterol diet; or $(c) 1 \%$ cholesterol diet supplemented by 2.25\% L-arginine $\mathbf{H C l}$ in drinking water. Arginine supplementation doubled plasma arginine levels without affecting serum cholesterol values. After 10 wk, the thoracic aorta was harvested for studies of vascular reactivity and histomorphometry.

Endothelium-dependent relaxations (to acetylcholine and calcium ionophore A23187) were significantly impaired in thoracic aortae from animals fed a $1 \%$ cholesterol diet. By contrast, vessels from hypercholesterolemic animals receiving $L$ arginine supplementation exhibited significantly improved endothelium-dependent relaxations. Responses to norepinephrine or nitroglycerin were not affected by either dietary intervention. Histomorphometric analysis revealed a reduction in lesion surface area and intimal thickness in thoracic aortae from arginine-supplemented animals compared to those from untreated hypercholesterolemic rabbits.

This is the first study to demonstrate that supplementation of dietary L-arginine, the EDRF precursor, improves endothelium-dependent vasorelaxation. More importantly, we have shown that this improvement in EDRF activity is associated with a reduction in atherogenesis. (J. Clin. Invest. 1992. 90:1168-1172.) Key words: endothelium-derived relaxing factor $\bullet$ nitric oxide • atherosclerosis • vasodilation • cholesterol
\end{abstract}

\section{Introduction}

Atherogenesis is a complex process that appears to be initiated by an endothelial injury or alteration (1). Elevated serum cholesterol, specifically the low density lipoprotein fraction, appears to play a major role in the process. One of the first endothelial alterations induced by LDL cholesterol is an attenuation of endothelium-dependent vasodilation (2-4). This endothelial dysfunction occurs before any pathological evi-

Address correspondence to John P. Cooke, M.D., Ph.D., Division of Cardiovascular Medicine, Stanford University School of Medicine, 300 Pasteur Drive, CVRC, Stanford, CA 94305.

Received for publication 18 February 1992 and in revised form 15 May 1992.

J. Clin. Invest.

(C) The American Society for Clinical Investigation, Inc.

0021-9738/92/09/1168-1172/05 \$2.00

Volume 90, September 1992, 1168-1172 dence of atherogenesis. Indeed, isolated vessels from normal animals manifest a reduction in endothelium-dependent vasodilation within minutes of exposure to oxidized LDL (5-6). This phenomenon appears to be caused by a reduced synthesis and/or increased degradation of endothelium-derived relaxing factor (EDRF) $)^{1}(7-12)$.

EDRF is now known to be nitric oxide or a molecule elaborating nitric oxide, and is derived from the metabolism of L-arginine (13-15). This endogenous nitrovasodilator also inhibits platelet adherence and aggregation (16-18), smooth muscle proliferation (19), and endothelial cell-leukocyte interactions $(20,21)$, all of which are key events in atherogenesis. For this reason, one might speculate that the reduction in EDRF activity induced by hypercholesterolemia may be an important endothelial alteration contributing to the atherogenic process.

We hypothesized that atherogenesis may be inhibited by chronically restoring EDRF activity in hypercholesterolemic animals. We have previously shown that intravenous administration of pharmacologic doses of $\mathrm{L}$-arginine could acutely improve endothelium-dependent vasodilation $(7,8,10)$. The present study was designed to determine if long-term, modest supplementation of dietary arginine could improve endothelium-dependent vasodilation, and to determine if this improvement in EDRF activity would be associated with an antiatherogenic effect.

\section{Methods}

\section{Animals}

Male New Zealand White rabbits $(n=54)$ were assigned to one of three treatment groups: 16 were fed normal rabbit chow for $10 \mathrm{wk}$ (Control); 18 received chow enriched with $1 \%$ cholesterol $(\mathrm{Chol})$; and 20 received $1 \%$ cholesterol diet supplemented with $2.25 \%$ L-arginine $\mathrm{HCl}$ in the drinking water ( $\mathrm{Arg}$ ) throughout the course of the study. Normal rabbit chow contains $0.9 \%$ L-arginine by weight. Supplementation with $2.25 \% \mathrm{~L}$-arginine $\mathrm{HCl}$ in drinking water results in a sixfold increase in daily L-arginine intake (based on the average water and food intake of the animals). This amount of L-arginine supplementation was chosen after preliminary studies demonstrated that this dose was well tolerated and resulted in a twofold increase in plasma free arginine levels $(0.32 \pm 0.02$ vs $0.16 \pm 0.02 ; \operatorname{Arg}(n=3)$ vs Control $(n=6))$.

After $10 \mathrm{wk}$ of the dietary intervention, animals were lightly sedated with acepromazine maleate solution (Ayerst Laboratories, New York) $3 \mathrm{mg}$ subcutaneous injection and the central ear arteries were cannulated for measurement of intraarterial blood pressure, followed by collection of blood samples. Blood pressure was measured through the arterial cannula after the animal was allowed to rest quietly for a mini-

1. Abbreviations used in this paper: EDRF, endothelial-derived relaxing factor; PSS, physiological saline solution. 
mum of $10 \mathrm{~min}$. Subsequently, the animals were killed and the thoracic aortae were harvested for studies of vascular reactivity and histology. These protocols were approved by the Administrative Panel on Laboratory Animal Care of Stanford University and were performed in accordance with the recommendations of the American Association for the Accreditation of Laboratory Animal Care.

\section{Biochemical studies}

Before the initiation of the diet, and after 5 and $10 \mathrm{wk}$ of treatment, blood samples were collected from animals in each group for measurement of plasma free arginine and serum chemistries, including total cholesterol. Plasma was deproteinized with $2 \%$ sulfosalicylic acid and analyzed for free arginine with an automated amino acid analyzer (Model 6300; Beckman Instruments Inc.; Spinco Division, Palo Alto, CA), as described previously (22-24). Serum chemistries were performed on an automated analyzer (Monarch Chemistry System; Instrumentation Laboratory, Inc., Lexington, MA).

\section{Vascular reactivity studies}

After harvesting the thoracic aortae, the tissues were placed into warm $\left(37^{\circ} \mathrm{C}\right)$ oxygenated physiological saline solution (PSS) of the following composition $(\mathrm{mM}): \mathrm{NaCl} 118.3, \mathrm{KCl} 4.7, \mathrm{CaCl}_{2} 2.5, \mathrm{MgSO}_{4}$ 1.2, $\mathrm{KH}_{2} \mathrm{PO}_{4} 1.2, \mathrm{NaHCO}_{3} 25$, calcium disodium edetate 0.026 , and glucose 11.1. The vessels were cleaned of adherent connective tissue, and the aortae were cut into rings ( $4 \mathrm{~mm}$ in length) for study in the organ chamber. The vascular rings were then suspended in organ chambers filled with $25 \mathrm{ml}$ PSS $\left(37^{\circ} \mathrm{C}\right.$ aerated with $\left.95 \% \mathrm{O}_{2} / 5 \% \mathrm{CO}_{2}\right)$. Rings were connected to force transducers, and changes in isometric force were recorded continuously. During a 60 -min period, the vascular rings were stretched to the optimal point of their length-tension relation (determined previously to be $4 \mathrm{~g}$ ). Subsequently, in all rings, the $\mathrm{EC}_{50}$ (concentration of drug inducing the half-maximal response) to norepinephrine was determined by exposing the tissues to increasing concentrations of norepinephrine added to the organ chamber in half-log increments in a cumulative manner. After a maximal response to norepinephrine was obtained, the vascular rings were washed repeatedly with fresh PSS until tension returned to the previous baseline value. To study vasodilating agents, rings were then contracted by the $\mathrm{EC}_{50}$ concentration of norepinephrine. After a stable contraction was obtained, the tissue was exposed to cumulative increases in concentration of the vasodilator in half-log increments.

\section{Histomorphometry}

Measurement of lesion surface area. In six animals from each group, thoracic aortae were harvested (from the left subclavian artery to the diaphragm). Adventitial tissue was dissected free and any remaining blood was rinsed clean. Aortae were bisected longitudinally and placed on a glass plate for photography of intimal lesions before and after oil-red $\mathrm{O}$ staining. These samples were stained in oil-red $\mathrm{O}(0.3 \% \mathrm{wt} /$ $\mathrm{vol}$ ) in $60 \%$ ( vol $/ \mathrm{vol}$ ) isopropyl alcohol for $5 \mathrm{~min}$, rinsed in $60 \%$ isopropyl alcohol, and then stained for 1 min in Harris' hematoxylin solution. Measurements of lesion surface area and total aortic surface area were made by planimetry of the photographic images.

Measurement of intimal thickness. In some animals, measurements of intimal and medial cross-sectional areas were made on segments taken from three sites in the thoracic aorta defined as follows: (a) A 5-mm segment proximal to the left subclavian artery; (b) a 5-mm segment equidistant from the left subclavian artery and the diaphragm; and (c) a 5-mm segment at the level of the diaphragm (proximal to the celiac artery). These three segments are referred to as the proximal, middle, and distal thoracic aorta, respectively. The segments were fixed in $10 \%$ buffered formalin, embedded in paraffin, sectioned, and stained with an elastic Van Giesen stain for light microscopy and histomorphometric measurements. Measurements of intimal and medial cross-sectional areas $\left(\mathrm{mm}^{2}\right)$ were made by a skilled observer blinded to the treatment groups. An eyepiece grid with 100 counting points (line intersections), each $0.4 \mathrm{~mm}$ apart, was used $(25,26)$. Total grid area $\left(\mathrm{mm}^{2}\right)$ was determined by measurement of grid dimensions with a stage micrometer at the same microscope magnification used for point counts. Total area of the intima (or media) was defined as the number of grid points overlying the intima (or media). Area of each was calculated by the formula $0.01(\mathrm{P}) \times$ grid area, where $\mathrm{P}$ is the number of points counted over the intima (or media). Six cross-sections from each vascular segment were analyzed, and the values were averaged.

\section{Drugs}

The drugs used were acetylcholine chloride, L-arginine hydrochloride, norepinephrine bitartrate, calcium ionophore A23187, (Sigma Chemical Co., St. Louis, MO), and nitroglycerin (DuPont Pharmaceuticals, Inc., Wilmington, DE). Drugs were dissolved in distilled water except for calcium ionophore A23187, which was dissolved in 2\% DMSO (Sigma). All drug solutions were prepared on the day of the experiment and stored on ice until use. Drugs were added to the organ chambers in volumes of $<150 \mu \mathrm{l}$. Concentrations are expressed as the final molar concentration in the bath solution.

\section{Data analysis}

Data are expressed as mean \pm SEM. Dose-response curves were constructed by expressing contractions as a percentage of the maximal response to norepinephrine; relaxations were expressed as a percentage of the tension induced by exposure to norepinephrine $\left(\mathrm{EC}_{50}\right.$ dose $)$. Each dose-response curve was characterized by determining the $\mathrm{EC}_{50}$ value (the dose of the drug inducing a half-maximal response), and by determining the maximal response to the drug. Multiple comparisons were avoided by comparing only the $\mathrm{EC}_{50}$ and maximal responses. Comparisons between two means were analyzed using the Student's unpaired $t$ test; $P<0.05$ was considered significant. Measurements of intimal thickness were analyzed using a multivariate analysis of variance to determine if there was an overall difference between the Chol and Arg groups. A modified Bonferonni correction was made for multiple comparisons between the groups.

\section{Results}

Biochemical and physiological measurements. There was a twofold elevation in plasma arginine levels in hypercholesterolemic animals maintained on oral L-arginine supplementation $(\mathrm{Arg})$ in comparison to animals on a normal (Control) or $1 \%$ cholesterol ( $\mathrm{Chol})$ diet alone; the elevation in plasma arginine was maintained throughout the course of the study (Fig. 1). Serum cholesterol measurements were elevated equally in both groups receiving the $1 \%$ cholesterol diet, and were both significantly different $(P<0.001$ for both comparisons) from animals receiving normal chow $(50 \pm 6$ vs. $1629 \pm 422$ vs.

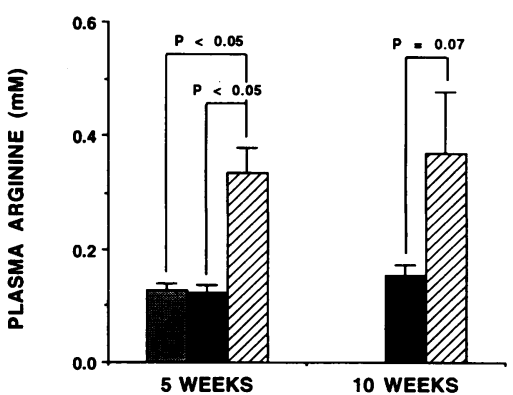

Figure 1. Plasma arginine values. Measurements were made in animals fed a normal diet, a $1 \%$ cholesterol diet, or a $1 \%$ cholesterol diet supplemented with L-arginine (Control, Chol, and Arg groups, respectively). L-arginine supplementation (2.25\% L-arginine $\mathrm{HCl}$ in drinking water) elevated the plasma concentration of this amino acid; the elevation was sustained throughout the course of the study. Measurements were made during weeks 5 and 10 of the dietary intervention. $n=6$ in each group. $\square$, Control; $\square \mathrm{Chol}$; Arg. 
$1852 \pm 356 \mathrm{mg} / \mathrm{dl}$, respectively for Control $(n=10)$, Chol $(n$ $=13)$, and $\operatorname{Arg}(n=14)$. After 10 wk of treatment, hypercholesterolemic animals receiving L-arginine supplementation had slightly elevated blood urea nitrogen measurements ( $14.6 \pm 1.2$ vs. $17.0 \pm 1.8$ vs $22.3 \pm 1.2 \mathrm{mg} / \mathrm{dl}$, respectively, for Control, Chol, and Arg; $P<0.05$, Arg vs. other groups).

There were no significant differences in hemodynamic measurements between the groups, although both groups receiving the $1 \%$ cholesterol diet tended to have elevated mean arterial pressures $(73 \pm 9$ vs. $89 \pm 7$ and $91 \pm 4 \mathrm{mmHg}$ in the Control $(n$ $=4)$, Chol $(n=9)$, and $\operatorname{Arg}(n=12)$ groups, respectively).

\section{Studies of vascular reactivity}

Endothelium-independent responses. The maximal tension induced by norepinephrine was not different between the groups, with $15.1 \pm 1.1,15.0 \pm 0.7$, and $13.9 \pm 0.6 \mathrm{~g}$ for Control, Chol, and Arg animals, respectively. The sensitivity to norepinephrine was not different between the groups, with $\mathrm{EC}_{50}(-\log \mathrm{m})$ values of $6.5 \pm 0.1,6.1 \pm 0.1$, and $6.0 \pm 0.1$, respectively for Control, Chol, and Arg animals. Similarly, there were no differences between the groups in sensitivity or maximal response to nitroglycerin with $\mathrm{EC}_{50}$ values of $7.8 \pm 0.1,7.5 \pm 0.1$, and $7.6 \pm 0.1$, and maximal vasodilation of $98 \pm 3.1,91 \pm 3.4$, and $84 \pm 4.6$ for Control, Chol, and Arg, respectively.

Endothelium-dependent responses. A multivariate analysis of variance revealed a significant difference between the groups in maximal relaxation to acetylcholine $(P<0.0003)$. Post-hoc unpaired comparisons between the groups revealed the maximal response to acetylcholine in the Chol group to be significantly less than that of both the Arg and the Control groups ( $61 \pm 5$ vs. $73 \pm 3$ vs. $89 \pm 2 \%$, Chol vs. Arg vs. Control, respectively; $P<0.05$; (Fig. 2). Whereas the maximal response to ACh of the Arg group was significantly greater than that of the Chol group, it was also less than that of the Control group ( $P$ $<0.05)$. There were no differences between the groups in sensitivity to $\mathrm{ACh}$, with $\mathrm{EC}_{50}$ values of $7.1 \pm 0.2,7.4 \pm 0.1$, and $7.5 \pm 0.1$ (Chol, Arg, and Control, respectively).

The maximal relaxation to calcium ionophore was not different between the groups, with maximal responses of $73 \pm 1.8$, $76 \pm 4.0$, and $87 \pm 3.5$ (Chol vs. Arg vs. Control, respectively; $P$ $<0.01)$. Post-hoc unpaired comparisons between the groups revealed that the sensitivity to calcium ionophore in the Chol group was significantly less than that of both the Arg and the Control groups, with $\mathrm{EC}_{50}$ values of $6.8 \pm 0.1,7.6 \pm 0.1$, and $7.4 \pm 0.3$ (Chol vs. Arg vs. Control, respectively; $P<0.05$ ).

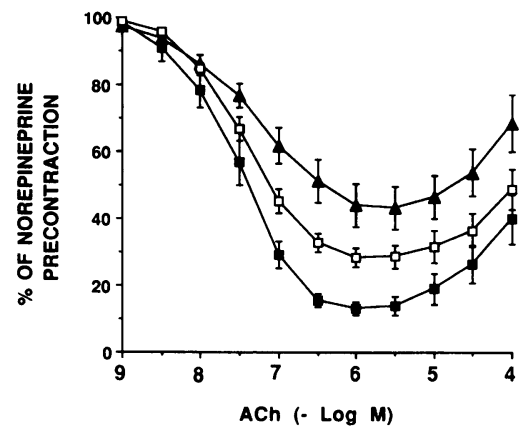

Figure 2. Endotheliumdependent relaxation in thoracic aortae. Endothelium-dependent relaxation to acetylcholine is reduced in the untreated hypercholesterolemic animals. The responsiveness to this agonist is significantly improved in the Arg group, although some residual impairment is retained. $-\square-$, Control; $-\Delta-$, Chol; - Arg.

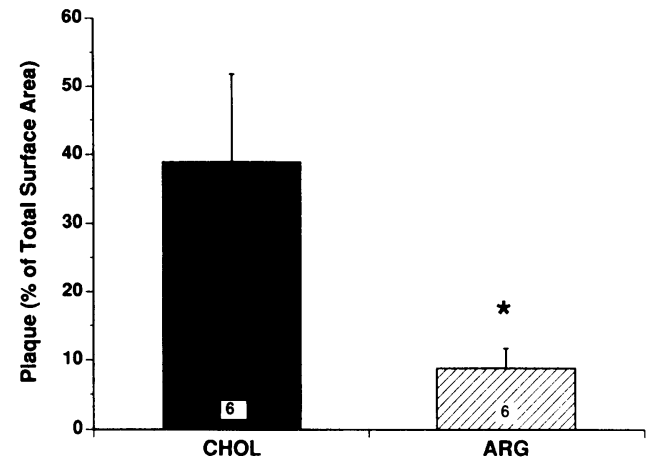

Figure 3. Planimetry of lesion surface area. The descending thoracic aortae from untreated hypercholesterolemic animals ( $\mathrm{Chol})$ and arginine-supplemented hypercholesterolemic animals $(\mathrm{Arg})$ were photographed for planimetry before and after oil-red $\mathrm{O}$ staining to assess intimal lipid accumulation. Lesion surface area was significantly reduced in the Arg group $(P<0.05)$. The numbers in the bars refer to the number of animals in each group.

\section{Histologic studies}

Surface area of intimal lesions. The thoracic aortae of control animals $(n=6)$ had no evidence of atheromatous lesions by visual inspection before or after staining with oil-red $\mathrm{O}$. Planimetric analysis by a blinded observer revealed that in the hypercholesterolemic animals $(n=6), \sim 40 \%$ of the surface area of the thoracic aorta was affected by intimal lipid accumulation (Fig. 3). By contrast, in the arginine-treated hypercholesterolemic animals $(n=6),<10 \%$ of the thoracic aorta was affected.

Intimal thickness. A blinded histomorphometric analysis revealed that medial cross-sectional areas were not different between the groups (data not shown). By contrast, the intimal cross-sectional area of vessels from hypercholesterolemic animals receiving $\mathrm{L}$-arginine supplementation was reduced in comparison to those from animals receiving the cholesterol diet alone. The reduction in intimal thickness in the Arg group was most pronounced in the distal thoracic aorta (with reductions in intimal thickness of $45 \%, 64 \%$, and $86 \%$ in the proximal, mid, and distal thoracic aorta, respectively; Fig. 4).

\section{Discussion}

This study provides the first evidence that the EDRF precursor, L-arginine, possesses antiatherogenic properties. In this study, chronic oral administration of modest doses of L-arginine to hypercholesterolemic rabbits decreased the surface area and reduced intimal thickness of atheromatous lesions in the tho-

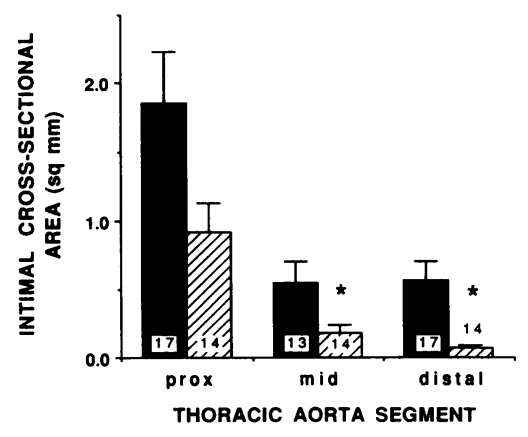

Figure 4. Histomorphometric measurements. Multivariate analysis of variance reveals an overall difference between the groups $(P$ $<0.05$ ). Unpaired $t$ tests with a modified Bonferonni correction were performed between the groups. Prox, proximal; mid, middle. -, Chol; , Arg. 
racic aorta. This antiatherogenic effect was associated with an improvement in endothelium-dependent relaxation, in the absence of any change in serum cholesterol. We propose that this normalization of vascular reactivity is caused by increased synthesis and release of EDRF. We further propose that this augmentation of EDRF activity is responsible for the antiatherogenic effect.

EDRF is not only a potent vasodilator, but also has inhibitory effects on platelet adherence and aggregation, as well as vascular smooth muscle proliferation (16-19). Nitric oxide also inhibits leukocyte-endothelial cell interactions. After reperfusion injury, sodium nitrite inhibits adherence and infiltration of neutrophils into the vessel wall (20). Monocyte adherence to cultured endothelial cells is also inhibited by nitric oxide (27). These phenomena may be caused by a direct effect of nitric oxide upon expression of glycoprotein adhesion molecules mediating endothelial cell-leukocyte interaction (21). Interaction of the vessel wall with circulating blood elements and disturbances of vascular smooth muscle growth are key events in atherogenesis. Our study suggests that endothelium-derived nitric oxide plays a major role in regulating these processes. However, this study does not exclude the possibility that nitric oxide derived from other cells may be contributing to the antiatherogenic effect. It is also possible that the effect of L-arginine on vascular structure is mediated by direct actions upon monocyte adherence or infiltration, platelet reactivity, or other key processes in atherogenesis. Nevertheless, our observation that L-arginine supplementation has antiatherogenic effects is novel, provocative, and of potential clinical importance.

We have heretofore demonstrated that acute intravenous administration of $\mathrm{L}$-arginine improves endothelium-dependent vasodilation in hypercholesterolemic animals and man. These effects were induced by pharmacological doses of the amino acid ( $10 \mathrm{mg} / \mathrm{kg}$ per min during $40 \mathrm{~min}$ ) that elevated plasma arginine levels 30 -fold. The present study reveals that chronic oral administration of modest doses of $\mathrm{L}$-arginine achieves a similar improvement in endothelial response with only a twofold increase in plasma arginine levels. Indeed, in preliminary studies $(n=3)$ it appears that a much smaller increment in dietary $\mathrm{L}$-arginine $(0.45 \% \mathrm{~L}$-arginine in drinking water rather than the $2.25 \%$ used in the present study) during a 10-wk course is sufficient to induce this effect. Chronic alterations in dietary arginine of this magnitude should be achieveable in man; studies are underway to determine if such a dietary intervention will induce similar augmentation of endothelial response in hypercholesterolemic human subjects.

We previously investigated the mechanism by which L-arginine improves endothelium-dependent vasodilation. Intravenous administration of L-arginine augments hind limb blood flow in response to acetylcholine in hypercholesterolemic rabbits (7). Similarly, thoracic aortae isolated from hypercholesterolemic rabbits receiving intravenous $\mathrm{L}$-arginine exhibit an improvement in endothelium-dependent relaxation in vitro (8). In both cases, the intravenous arginine infusion did not alter endothelium-independent responses to sodium nitroprusside. Thus, the beneficial effect of $L$-arginine is not caused by a direct action on vascular smooth muscle relaxation. Furthermore, this effect is stereo-specific, since $\mathrm{D}$-arginine did not mimic the action of its enantiomer in either study $(7,8)$. Administration of L-arginine has other systemic consequences, most notably its ability to release insulin from pancreatic islet cells (28). However, we have shown that endothelium-depen- dent vasodilation can be improved in basilar arteries isolated from hypercholesterolemic rabbits and perfused in vitro with solutions containing $\mathrm{L}$-arginine. These data provide strong evidence against other in vivo effects of the amino acid as an explanation for its action (9). A similar effect of $\mathrm{L}$-arginine to reduce the endothelial dysfunction associated with reperfusion injury has recently been reported (29).

Vascular rings from normal rabbits exposed in vitro to oxidized low density lipoprotein rapidly develop an endothelial impairment that is reversible by the addition of $\mathrm{L}$-arginine to the bath solution (30), a finding that is consonant with our previous studies in hypercholesterolemic animals and in man. By contrast, Mügge and Harrison find that the endothelial dysfunction manifested by atherosclerotic rabbit aorta is not reversible in vitro (31). An important difference in their experimental model is the length of cholesterol feeding ( $4 \mathrm{mo}$ ), by which time the thoracic aorta demonstrates significant pathology. The opposing conclusion derived from this study may be reconciled if the reversibility of endothelial dysfunction is dependent upon the chronicity or severity of the disease process. A selective dysfunction in endothelial response is observed early in hypercholesterolemic states. Later, endothelium-dependent relaxation to most stimuli becomes progressively abnormal $(32,33)$. Evidence that the reversibility of endothelial dysfunction depends on the degree of attendant vascular pathology may be found in a recent report by Drexler et al. (34). These investigators observed that acetylcholine-induced vasodilation of epicardial coronary arteries and coronary resistance vessels was attenuated in hypercholesterolemic human subjects. After intracoronary administration of L-arginine, there was no improvement in epicardial coronary artery response. In contrast, there was a marked improvement in coronary blood flow response to acetylcholine, reflecting an improvement in endothelial function at the level of the resistance vessels. We have observed the same phenomenon in the coronary circulation of cardiac transplant recipients (unpublished studies). This difference in response to arginine might be explained by the observation that hypercholesterolemia induces greater endothelial pathology in the conduit arteries than the resistance vessels.

In the present study we tested the hypothesis that a chronic augmentation of EDRF activity in hypercholesterolemic animals would have antiatherogenic effects. We show that modest supplementation of dietary arginine improves endotheliumdependent vasorelaxation. This improvement in EDRF activity is associated with a reduction in lesion surface area and intimal thickening in hypercholesterolemic animals. The mechanisms of these intriguing phenomena remain to be fully elucidated.

\section{Acknowledgments}

Plasma arginine levels were performed with the technical assistance of Alan J. Smith and James W. Kenny of the Protein and Nucleic Acid Facility at Stanford University. Biostatistical assistance was provided by Jerry Halpern of the Department of Biostatistics of Stanford University.

This work was supported by a grant-in-aid from the Institute of Biological and Clinical Investigation of Stanford University, as well as a grant-in-aid from the Erario Fund. Dr. Singer is the recipient of an American Heart Association-Bugher Fellowship. Dr. Cooke is a recipient of the Vascular Academic Award from the National Heart, Lung, and Blood Institute 1KO7HCO2660-01. 


\section{References}

1. Ross, R. 1986. The pathogenesis of atherosclerosis. N. Engl. J. Med. 314:488-500.

2. Verbeuren, T. J., F. H. Jordaens, L. L. Zonnekeyn, C. E. VanHove, M. C. Coene, and A. G. Herman. 1986. Effect of hypercholesterolemia on vascular reactivity in the rabbit. Circ. Res. 58:552-564.

3. Cohen, R. A., K. M. Zitnay, C. C. Haudenschild, and L. D. Cunningham. 1988. Loss of selective endothelial cell vasoactive functions caused by hypercholesterolemia in pig coronary arteries. Circ. Res. 63:903-910.

4. Ludmer, P. L., A. P. Selwyn, T. L. Shook, R. R. Wayne, G. H. Mudge, R. W. Alexander, and P. Ganz. 1986. Paradoxical vasoconstriction induced by acetylcholine in atherosclerotic coronary arteries. N. Engl. J. Med. 315:10461051 .

5. Andrews, H. E., K. R. Bruckdorfer, R. C. Dunn, and M. Jacobs. 1987. Low-density lipoproteins inhibit endothelium-dependent relaxation in rabbit aorta. Nature (Lond.). 327:237-239.

6. Kugiyama, K., S. A. Kerns, J. D. Morrisett, R. Roberts, and P. D. Henry. 1990. Impairment of endothelium-dependent arterial relaxation by lysolecithin in modified low-density lipoproteins. Nature (Lond.). 344:160-162.

7. Girerd, X. J., A. T. Hirsch, J. P. Cooke, V. J. Dzau, and M. A. Creager 1990. L-arginine arguments endothelium-dependent vasodilation in cholesterolfed rabbits. Circ. Res. 67:1301-1308.

8. Cooke, J. P., N. A. Andon, X. J. Girerd, A. T. Hirsch, and M. A. Creager. 1991. Arginine restores cholinergic relaxation of hypercholesterolemic rabbit thoracic aorta. Circulation. 83:1057-1062.

9. Rossitch, E., Jr., E. Alexander III, P. Black, and J. P. Cooke. 1991. L-arginine normalizes endothelial function in cerebral vessels from hypercholesterolemic rabbits. J. Clin. Invest. 87:1295-1299.

10. Creager, M. A., S. Gallagher, X. J. Girerd, V. J. Dzau, and J. P. Cooke. 1990. L-arginine normalizes endothelial response in the forearm circulation of hypercholesterolemic humans. Circulation. 82(Suppl. III):III-346. (Abstr.)

11. Minor, R. L., Jr., P. R. Myers, R. Guerra, Jr., J. N. Bates, and D. G. Harrison. 1990. Diet-induced atherosclerosis increases the release of nitrogen oxides from rabbit aorta. J. Clin. Invest. 86:2109-2116.

12. Chin, J. H., S. Azhar, and B. B. Hoffman. 1992. Inactivation of endothelial derived relaxing factor by oxidized lipoproteins. J. Clin. Invest. 89:10-18.

13. Palmer, R. M. J., A. G. Ferridge, and S. Moncada. 1987. Nitric oxide release accounts for the biological activity of endothelium-derived relaxing factor. Nature (Lond.). 327:524-526.

14. Ignarro, L. J., R. E. Byrns, G. M. Buga, and K. S. Wood. 1987. Endothelium-derived relaxing factor from pulmonary artery and vein possesses pharmacologic and chemical properties identical to those of nitric oxide radical. Circ. Res. 61:866-879.

15. Palmer, R. M. J., D. S. Ashton, and S. Moncada. 1988. Vascular endothelial cells synthesize nitric oxide from L-arginine. Nature (Lond.). 333:664-666.

16. Radomski, M. W., R. M. J. Palmer, and S. Moncada. 1987. Comparative pharmacology of endothelium-derived relaxing factor, nitric oxide, and prostacyclin in platelets. Br. J. Pharmacol. 92:181-187.

17. Stamler, J. S., M. E. Mendelsohn, P. Amarante, D. Smick, N. Andon, P. F. Davies, J. P. Cooke, and J. Loscalzo. 1989. $\mathrm{N}$-acetylcysteine potentiates platelet inhibition by endothelium-derived relaxing factor. Circ. Res. 65:789-795.
18. Radomski, M. W., R. M. J. Palmer, and S. Moncada. 1990. An L-arginine/nitric oxide pathway present in human platelets regulates aggregation. Proc. Natl. Acad. Sci. USA. 87:5193-5197.

19. Garg, U. C., and A. Hassid. 1989. Nitric oxide-generating vasodilators and 8-bromo-cyclic guanosine monophosphate inhibit mitogenesis and proliferation of cultured rat vascular smooth muscle cells. J. Clin. Invest. 83:1774-1777.

20. Johnson, G., P. S. Tsao, D. Malloy, and A. M. Leffer. 1990. Cardioprotective effects of acidified sodium nitrite in myocardial ischemia with reperfusion. $J$. Pharmacol. Exp. Ther. 252:35-41.

21. Kubes, P., M. Suzuki, and D. N. Granger. 1991. Nitric oxide: an endogenous modulator of leukocyte adhesion. Proc. Natl. Acad. Sci. USA. 88:46514655

22. Spackman, D. H., W. H. Stein, and S. Moore. 1958. Automatic recording apparatus for use in chromatography of amino acids. Anal. Chem. 30:1190.

23. Smoyer, W. E., B. H. Brouhard, D. K. Rassin, and L. LaGrone. 1991 Enhanced GFR response to oral versus intravenous arginine administration in normal adults. J. Lab. Clin. Med. 118:166-175.

24. Carey, J. P., K. Zane, et al. 1987. An arginine-deficient diet in humans does not evoke hyperammonemia or orotic aciduria. J. Nutr. 117:1734-1739.

25. Aherne, M. A., and M. S. Dunnill. 1992. Morphometry. Edward Arnold, Sevenoaks, Kent, England.

26. Manwaring, L., D. L. O'Connell, B. S. Bhagwandeen, I. M. Zardawi, and A. J. Dobson. 1988. Morphometric analysis of coronary artery stenosis: an accuracy and reliability study. J. Pathol. 156:111-117.

27. Bath, P. M., D. G. Hassall, A. M. Gladwin, R. M. Palmer, and J. F. Martin. 1991. Nitric oxide and prostacyclin. Divergence of inhibitory effects on monocyte chemotaxis and adhesion to endothelium in vitro. Arterioscler. Thromb. 11:254-260.

28. Schmidt, H. H., T. D. Warner, K. Ishii, H. Sheng, and F. Murad. 1992. Insulin secretion from pancreatic B cells caused by L-arginine-derived nitrogen oxides. Science (Wash. DC). 255(5045):721-723.

29. Nakanishi, K., J. Vinten-Johansen, D. J. Lefer, W. C. Fowler III, W. E Johnston, D. S. McGee, and B. Gray. 1992. Intracoronary L-arginine infusion during reperfusion reduces infarct size and neutrophil accumulation. Scientific Conference on Functional and Structural Mechanisms of Vascular Control, Snowbird, Utah. p. 86. (Abstr.)

30. Tanner, F. C., G. Noll, C. M. Boulanger, and T. F. Lüscher. 1991. Oxidized low density lipoproteins inhibit relaxations of porcine coronary arteries. Role of scavenger receptor and endothelium-derived nitric oxide. Circulation. 83:2012-2020.

31. Mügge, A., and D. G. Harrison. 1991. L-arginine does not restore endothelial dysfunction in atherosclerotic rabbit aorta in vitro. Blood Vessels. 28:354357.

32. Shimokawa, H., and P. M. Vanhoutte. 1989. Impaired endothelium-dependent relaxation to aggregating platelets and related vasoactive substances in porcine coronary arteries in hypercholesterolemia and atherosclerosis. Circ. Res. 64:900-914.

33. Zeiher, A. M., H. Drexler, H. Wollschläger, and H. Just. 1991. Modulation of coronary vasomotor tone in humans. Progressive endothelial dysfunction with different early stages of coronary atherosclerosis. Circulation. 83:391-401.

34. Drexler, H., A. M. Zeiher, K. Meinzer, and H. Just. 1991. Correction of endothelial dysfunction in the coronary microcirculation of hypercholesterolemic patients by L-arginine. Lancet. 338:1546-1550. 